\title{
The '2014 European guideline on the management of syphilis' has now been published
}

M Unemo (magnus.unemo@orebroll.se) ${ }^{1,2}$, M Janier ${ }^{2,3}$

1. WHO Collaborating Centre for Gonorrhoea and other Sexually Transmitted Infections, Department of Laboratory Medicine, Microbiology, Örebro University Hospital, Örebro, Sweden

2. European STI Guidelines Editorial Board (www.iusti.org/regions/Europe/euroguidelines.htm)

3. STD Clinic, Hôpital Saint-Louis AP-HP and Hôpital Saint-Joseph, Paris, France

Citation style for this article:

Unemo M, Janier M. The '2014 European guideline on the management of syphilis' has now been published . Euro Surveill. 2014;19(45):pii=20957. Available online: http://www.eurosurveillance.org/ViewArticle.aspx?Articleld=20957

On 27 October 2014, the evidence-based '2014 European guideline on the management of syphilis' was published online [1]. This guideline, a thoroughly updated version of the 2008 version [2], provides upto-date guidance regarding the diagnosis and management of syphilis in Europe. It includes important updates such as:

- recommendations for broader use of validated and quality assured PCRs and new treponemal tests;

- more flexible options for serologic screening, that is, traditional sequence algorithm (non-treponemal test as initial test), reverse sequence algorithm (treponemal test as initial test), or a combined version (both non-treponemal and treponemal tests for ideal initial testing);

- discussions regarding advantages and disadvantages with different screening algorithms, including obtaining false-negative and false-positive specimens in the serologic diagnosis;

- recommending long-acting penicillin G (benzathine penicillin $\mathrm{G}$ ) as the only first-line therapy regimen in early syphilis and in late latent syphilis, that is, procaine penicillin is no longer any first-line therapy option in any phase of syphilis.

Further details regarding recommended diagnostics, when to use and how to interpret the results of different tests, recommended management of neurosyphilis and cardiovascular, ocular, auricular and congenital syphilis, alternative treatment regimens, contact tracing, management of sexual contacts, and follow-up including test-of-cure, are also available in the newly launched guideline [1].

Syphilis, classified as acquired or congenital, with the aetiological agent Treponema pallidum subspecies pallidum remains a major public health concern globally. In 2008, the World Health Organization (WHO) estimated 10.6 million cases among adults worldwide ( 0.2 million in the WHO European region) [3]. In the European Union and European Economic Areas, 20,802 syphilis cases were reported (5.1 [range: $1-8.5$ ] per 100,000 population) in 2012 [4]. The majority ( $>80 \%)$ of syphilis cases were reported in patients $\geq 25$ years of age and half $(48 \%)$ of the cases were reported in men who have sex with men (MSM). After a long-term decreasing trend, overall rates are now relatively stable but several countries have reported dramatic increases during last decade, mainly among MSM. Furthermore, 91 cases of congenital syphilis cases were reported in 11 countries (23 reporting countries) in 2012 [4].

As long as appropriate testing of suspicious syphilis cases and/or screening are performed, syphilis is relatively easy to detect by adequate serological tests, supplemented with a validated and quality assured PCR in particularly early primary syphilis. However, all laboratory results should be considered together with clinical data and sexual risk anamnesis. Syphilis is also easy to treat with benzathine penicillin G. Suboptimal supply of benzathine penicillin $\mathrm{G}$ in several European countries could threaten the management of syphilis, including the initiative to eradicate congenital syphilis, in Europe.

\section{* Author's correction:}

On 18 November 2014, the number of cases of congenital syphilis reported in 2012 was changed from 101 to 91 at the request of the authors.

\section{References}

1. Janier M, Hegyi V, Dupin N, Unemo M, Tiplica GS, Potočnik $M$, et al. 2014 European guideline on the management of syphilis. J Eur Acad Dermatol Venereol. 2014 Oct 27. Available from: http://www.iusti.org/regions/europe/pdf/2014/JEADV_ FINAL_28_10_2014.pdf

2. French $P$, Gomberg $M$, Janier $M$, Schmidt B, van Voorst Vader $P$, Young H. IUSTI: 2008 European guidelines on the management of syphilis. Int J STD AIDS. 2009;20(5):300-9.

3. World Health Organization (WHO). Global incidence and prevalence of selected curable sexually transmitted infections - 2008. Geneva: WHO; 2012. p. 1-28. Available from: http:// www.who.int/reproductivehealth/publications/rtis/2008_STI_ estimates.pdf

4. European Centre for Disease Prevention and Control (ECDC). 2014. Sexually Transmitted Infections in Europe 2012

Stockholm: ECDC; 2014. Available from: http://www.ecdc. europa.eu/en/publications/Publications/sexually-transmittedinfections-europe-surveillance-report-2012.pdf 